\title{
The Role of Cannabinoids in the Neurobiology of Sensory Gating: A firing rate model study
}

\author{
Margarita Zachariou ${ }^{a}$, Dilshani W. N. Dissanayake ${ }^{\mathrm{b}}$, \\ Markus R. Owen ${ }^{\mathrm{a}}$, Rob Mason ${ }^{\mathrm{b}}$ and Stephen Coombes ${ }^{\mathrm{a}}$ \\ ${ }^{a}$ School of Mathematical Sciences, University of Nottingham, Nottingham, NG7 \\ 2RD, $U K$ \\ ${ }^{\mathrm{b}}$ Neuronal Networks Electrophysiology Laboratory, School of Biomedical Sciences, \\ University of Nottingham, Nottingham, NG7 2UH, UK
}

\begin{abstract}
Gating of sensory (e.g. auditory) information has been demonstrated as a reduction in the auditory-evoked potential responses recorded in the brain of both normal animals and human subjects. Auditory gating is perturbed in schizophrenic patients and pharmacologically by drugs such as amphetamine, phencyclidine or ketamine, which precipitate schizophrenic-like symptoms in normal subjects. The neurobiological basis underlying this sensory gating can be investigated using local field potential recordings from single electrodes. In this paper we use such technology to investigate the role of cannabinoids in sensory gating. Cannabinoids represent a fundamentally new class of retrograde messengers which are released postsynaptically and bind to presynaptic receptors. In this way they allow fine-tuning of neuronal response, and in particular can lead to so-called depolarization-induced suppression of inhibition (DSI). Our experimental results show that application of the exogenous cannabinoid WIN55, 212-2 can abolish sensory gating as measured by the amplitude of local field responses in rat hippocampal region CA3. Importantly we develop a simple firing rate population model of $\mathrm{CA} 3$ and show that gating is heavily dependent upon the presence of a slow inhibitory $\left(\mathrm{GABA}_{\mathrm{B}}\right)$ pathway. Moreover, a simple phenomenological model of cannabinoid dynamics underlying DSI is shown to abolish gating in a manner consistent with our experimental findings.
\end{abstract}

Key words: Sensory gating, cannabinoids, depolarization-induced suppression of inhibition, firing rate models. 


\section{Introduction}

Sensory gating deficit is an attentional disorder, characterised by the inability to adequately filter incoming sensory information, which has been observed in schizophrenic patients [9]. A deficit in sensory gating can be demonstrated in human subjects using the ratio of P50 auditory-evoked responses to a conditioning testing paradigm, in which two auditory tones are presented $500 \mathrm{~ms}$ apart. The P50 auditory evoked potential is identified as the largest amplitude positive waveform recorded in the electroencephalogram (EEG) approximately 50ms after the auditory tone stimulus. Normal subjects have a smaller response to the second (test) tone than to the first (conditioning) tone. In contrast schizophrenic subjects fail to demonstrate a gated response to the second tone. The test response of normal controls is often less than $15 \%$ of their conditioning response, whereas the test response of schizophrenic subjects is often more than $85 \%$ of their conditioning response.[9] Therefore, the ratio of the amplitude of the testing to the conditioning response $(\mathrm{T} / \mathrm{C})$ is used as a quantitative measure to assess a person's ability to filter sensory information [9]. A $\mathrm{T} / \mathrm{C}$ ratio value below or equal to 0.5 has been considered to indicate normal sensory gating [13].

In the rat the equivalent of the human P50 wave is thought to be the N40 component of the EEG, a mid-latency negative evoked potential occurring approximately 40ms after an auditory stimulus tone $[3,13]$. Sensory gating can also be detected in the averaged local field potential (LFP) signal and in the single neuron activity from principal cells and interneurons in the CA3 hippocampal region. An example of sensory gating is illustrated in figure 1, showing an average auditory evoked LFP from a single electrode in the CA3 region of an anaesthetised rat [7].

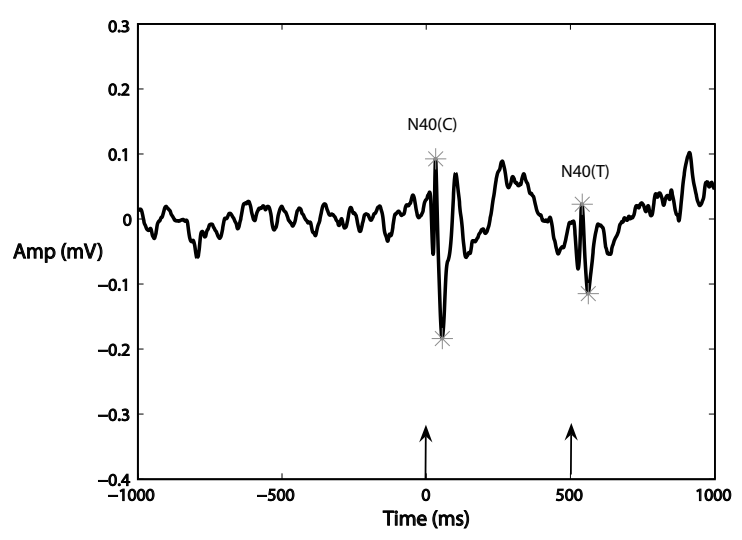

Fig. 1. Averaged auditory evoked LFP responses, recorded from the CA3 region of anaesthetised rat averaged over 128 trials. The two auditory $3 \mathrm{kHz}$ tones presented 500ms apart, are indicated by the arrows. The rat exhibits normal gating with $\mathrm{T} / \mathrm{C}=0.50$. Data from [7]. 


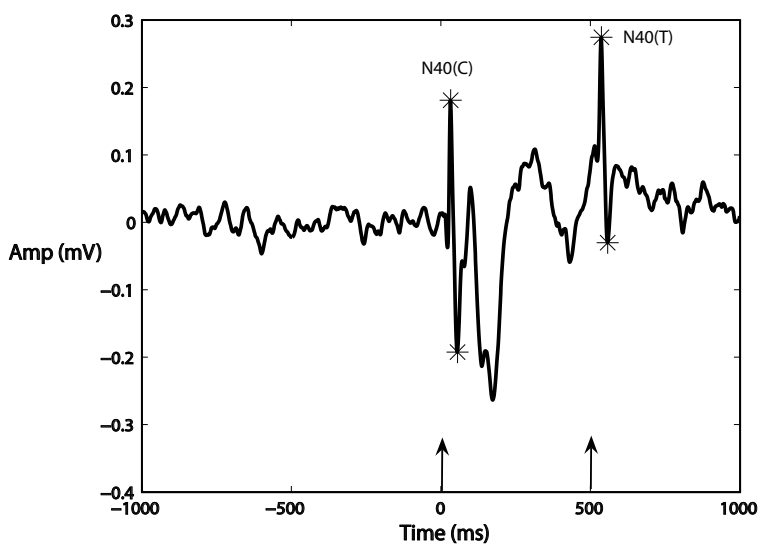

Fig. 2. After administration of non-selective cannabinoid agonist WIN55, 212-2, a loss of sensory gating is demonstrated, indicated by the high value of $\mathrm{T} / \mathrm{C}=0.82$. Data from [7].

The CA3 hippocampal region consists of two main neuronal populations; notably pyramidal cells which are the principal cells and have an excitatory action, and basket cells which are inhibitory and play the role of interneurons. It has been estimated anatomically that about $90 \%$ of the cells in the CA3 hippocampus region are pyramidal principal output neurons whose activity is excitatory, and the rest are inhibitory neurons, of which the majority is basket cells [9]. The neurotransmitters acetylcholine, dopamine and GABA have been suggested to play a role in sensory gating dysfunction. Experimental studies in rats have shown that blocking the acetylcholine (ACh) transmission either pharmacologically [17] or by lesioning the cholinergic input (neurons or synapses that produce and release ACh) [2] results in impairment of normal gating, which can be restored by administration of nicotine (mimicking the effects of ACh at nicotinic ACh receptors).

Previous studies have reported that amphetamine, a dopamine receptor agonist, can reduce sensory gating. Since this reduction is mediated by the decrease of the amplitude corresponding to the first auditory tone, there are doubts whether dopamine receptor activation affects the supposed inhibitory process between the first and the second tone [6,5]. Bickford-Wimer et al. (1990) [3] have shown that the amphetamine-induced reduction of gating in hippocampus (in rats) was antagonised by the dopamine receptor antagonist haloperidol. Further, it has been suggested that gating is lost due to a deficit in the inhibitory recurrent activity within the hippocampus $[13,12]$. A role for $\mathrm{GABA}_{\mathrm{B}}$ receptors in disrupting sensory gating has been demonstrated via the administration of $\mathrm{GABA}_{\mathrm{B}}$ antagonists [11]. Note that GABAergic inhibition might not be the only slow inhibitory process involved in sensory gating and a role has been proposed for the neuromodulator adenosine [12]. Other pre-synaptic modulators released by interneurons, such as somatostatin and neuropeptide Y, may also be involved in sensory gating [13].

Previous modelling studies have explored the local processing and afferent activity in- 
volvement in sensory gating $[9,15,14]$. Moxon et al. $[15,14]$ have explained the nicotinic cholinergic input role in sensory gating and the dopaminergic modulation of the P50 $(\mathrm{N} 40)$. In addition they have suggested that $\mathrm{GABA}_{\mathrm{B}}$ synapses are involved in suppressing the second (test) tone, by suppressing cortical input and recurrent excitation. They propose this inhibitory pathway is indirectly activated by nicotinic cholinergic input from the septum.

A 'cannabinoid hypothesis of schizophrenia' has been proposed, suggesting that the overactivity of endogenous cannabinoids ( $\mathrm{CBs}$ ) may be involved in the pathophysiology of schizophrenia [8]. Preliminary experimental results in our laboratory have shown that the administration of the non-selective $\mathrm{CB}_{1} / \mathrm{CB}_{2}$ cannabinoid agonist WIN55, 212-2 abolishes N40 gating in healthy rats [7] (see figure 2).

Endogenous cannabinoids represent a fundamentally new class of retrograde messengers [10], which are released postsynaptically and bind to presynaptic CB receptors. CB synthesis is stimulated when levels of calcium rise inside the neuron or when certain Gprotein-coupled receptors are activated. One function of endogenous CBs is to regulate neurotransmitter release via activation of presynaptic $\mathrm{CB}_{1}$ receptors. Activation of $\mathrm{CB}_{1}$ receptors on hippocampal interneurons reduces their release of the inhibitory neurotransmitter GABA. Retrograde signalling from a strongly depolarised postsynaptic cell to the presynaptic GABA-releasing cell to shut off GABA release is termed Depolarizationinduced Suppression of Inhibition (DSI) [19]. Recent studies support the view that DSI is mediated by CBs. It has been shown that hippocampal DSI was blocked by $\mathrm{CB}_{1}$ antagonists and can be mimicked by application of the $\mathrm{CB}_{1}$ receptor agonists $[20,16]$.

DSI, as a form of short-term synaptic plasticity of GABAergic transmission, has been suggested to facilitate a form of learning called long-term potentiation (LTP). This is the process by which information is stored through the strengthening of excitatory synapses. CB mediated DSI may promote strengthening of excitatory synapses (LTP) by blocking inhibition of principal cells [19]. We suggest that the CB-mediated transient reduction in inhibition associated with DSI could be linked to the sensory gating deficit. Altering the activity of the cannabinoid system (which may be inactive under normal basal circumstances [4]) by recreational drug abuse or as a result of pathological conditions, such as schizophrenia, could impair sensory gating through suppression of inhibition from interneurons.

In this paper we develop a mathematical model for the basic local network mechanisms involved in sensory gating. We suggest that sensory gating can be explained by a slow mechanism (possibly representing $\mathrm{GABA}_{\mathrm{B}}$ receptor activity). Moreover we include $\mathrm{CB}$ mediated DSI via a phenomenological description of CB dynamics and examine the con- 
sequences for gating.

\section{The Model}

Here we develop a minimal firing rate model of the pyramidal and basket cells that form the bulk of the CA3 hippocampal network, by following the activity of fast AMPA excitatory synapses, fast $\mathrm{GABA}_{\mathrm{A}}$ inhibitory synapses and slow $\mathrm{GABA}_{\mathrm{B}}$ inhibitory synapses. We will show that this approach is sufficient to model the basic mechanisms underlying gating. Importantly this minimal approach can be naturally extended to include a phenomenological description of CB dynamics. The intrinsic network of the CA3 region in hippocampus is modelled using Wilson-Cowan style equations for neuronal population activity [18]. The activity of the populations is described by the following equations,

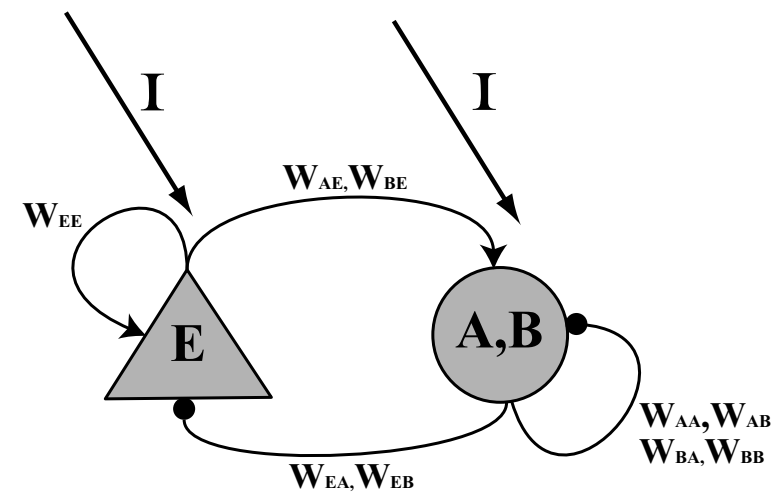

Fig. 3. Both populations receive sensory input (I) from the two auditory tones paradigm. The principal cells (E) excite themselves and the inhibitory population (A,B). The interneurons inhibit themselves and the principal cells with both slow and fast inhibition.

$$
\begin{aligned}
Q_{E} E & =f\left(W_{E A} A+W_{E B} B+W_{E E} E+I\right) \\
Q_{A} A & =f\left(W_{A A} A+W_{A B} B+W_{A E} E+I\right) \\
Q_{B} B & =f\left(W_{B A} A+W_{B B} B+W_{B E} E+I\right)
\end{aligned}
$$

where

$$
Q_{X}=\left(1+\alpha_{X}^{-1} \frac{\mathrm{d}}{\mathrm{d} t}\right)^{2},
$$

and $X \in\{A, B, E\}$. Here $E$ is the excitatory activity of the principal neuronal population, $A$ is the fast and $B$ the slow inhibitory activity respectively, of the interneurons. The variables $W_{X Y}$ represent the strength of connections from population $Y$ to population $X$. Excitatory synapses have a positive value $\left(W_{X E}>0\right)$, and inhibitory synapses have a negative value $\left(W_{X A}, W_{X B}<0\right)$. Note that in contrast to the standard Wilson-Cowan model we take $Q_{X}$ to be a second order, rather than first order, differential operator. In 
this way the synaptic response is modelled as an alpha-function of the form $\alpha_{X}{ }^{2} t \mathrm{e}^{-\alpha_{X} t} \Theta(t)$, where $\Theta(t)$ is a Heaviside step function (such that $\Theta(t)=1$ of $t \geq 0$ and is zero otherwise). Here $\alpha_{X}$ denotes the inverse rise and fall time of synaptic activity of the corresponding population $X$.

The external input is a simple two-tone temporal sequence of the form $I=\Theta(t-1000)-$ $\Theta(t-1010)+\Theta(t-1500)-\Theta(t-1510)$. The function $f$ is a firing rate which we take to be of the sigmoidal form $f=\sigma_{\beta}(u)$, where

$$
\sigma_{\beta}(u)=\frac{1}{1+e^{-\beta u}}
$$

Here $\beta$ parameter gives the steepness of the sigmoid function.

To model the effect of the $\mathrm{CB}$ mechanism in reducing the release of GABA from the inhibitory population, we choose $W_{E B}$ and $W_{E A}$, representing the GABAergic input to the principal population, to be reduced in the presence of either exogenous or endogenous CBs in the network. For simplicity we take $W_{E X}(C B)=\bar{W}_{E X}\left[1-\sigma_{\gamma}(C B)\right]$ for $X \in\{A, B\}$ with $\sigma_{\gamma}$ defined by (3) and

$$
C B=C B_{\text {exo }}+b C B_{\text {endo }},
$$

where $b \in\{0,1\}$. Here the labels exo and endo refer to exogenous and endogenous levels of $\mathrm{CB}$ respectively. We shall regard the former to be under experimental control and the latter to be a dynamical process that subserves DSI. A simple phenomenological model of this process can be written

$$
\tau \frac{\mathrm{d} C B_{\text {endo }}}{\mathrm{d} t}=-C B_{\text {endo }}+\sigma_{\delta}(E)
$$

Here $\tau$ is the degradation time course of the endogenous CBs.

LFPs are considered to represent the synchronised summed synaptic currents arising from the activity of large local neuronal populations [1]. In the absence of a physical model that quantitatively links LFPs to synaptic activities we simply take a measure of LFP in our framework as a linear combination of the population activities: $L=a_{1} E+a_{2} A+a_{3} B-L_{0}$ (where the subtraction of the term $L_{0}$ fixes the steady state activity in the absence of inputs to be $L=0$ ).

Finally, we note that the external inputs have the same amplitude. However, this does not reflect the fact that sensory input is filtered through the non-lemniscal pathway and that the signal corresponding to the test tone already arrives in the hippocampus gated to some degree [13]. Nevertheless, in order to emphasize the effect of the slow inhibitory process, we model the input as having the same amplitude for both tones and therefore do not expect to achieve the same degree of gating as in experiments. 
In figure 4 we plot the simulated LFP, $L$, when only endogenous CBs are present $\left(C B_{\text {exo }}=\right.$ 0 ). This demonstrates the ability of the model to gate sensory information, with a $\mathrm{T} / \mathrm{C}$ ratio of 0.83 . The basic mechanism for the reduction of the response to the test tone is the activation of the slow $\mathrm{GABA}_{\mathrm{B}}$ pathway.

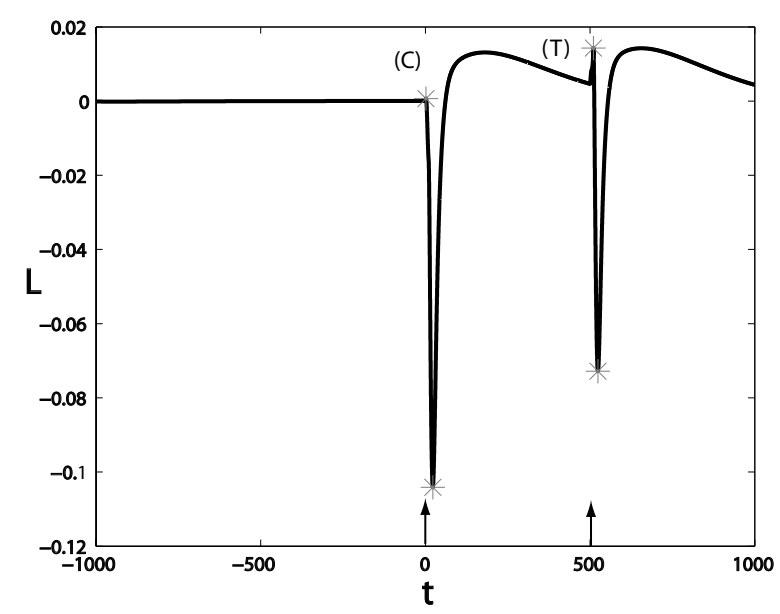

Fig. 4. Under basal conditions gating is observed with $\mathrm{T} / \mathrm{C}$ ratio $0.83 . W_{E E}=W_{A E}=W_{B E}=1$, $W_{A A}=W_{A B}=W_{B A}=W_{B B}=-1, \bar{W}_{E A}=-2, \bar{W}_{E B}=-20, \alpha_{E}=0.1, \alpha_{A}=0.2, \alpha_{B}=0.005$, $\beta=10, \gamma=\delta=1, \tau=100, C B_{\text {exo }}=0, b=1, a_{1}=-1, a_{2}=0.25, a_{3}=1$.

When the model includes exogenous $\mathrm{CBs}\left(C B_{\text {exo }} \neq 0\right)$ at sufficiently high levels gating is abolished, with a $\mathrm{T} / \mathrm{C}$ ratio of 1 , as seen in figure 5 . In essence this is due to the suppression of $\mathrm{GABA}_{\mathrm{B}}$ release that occurs when $\mathrm{CB}_{1}$ receptors are activated by $\mathrm{CB}$ agonists.

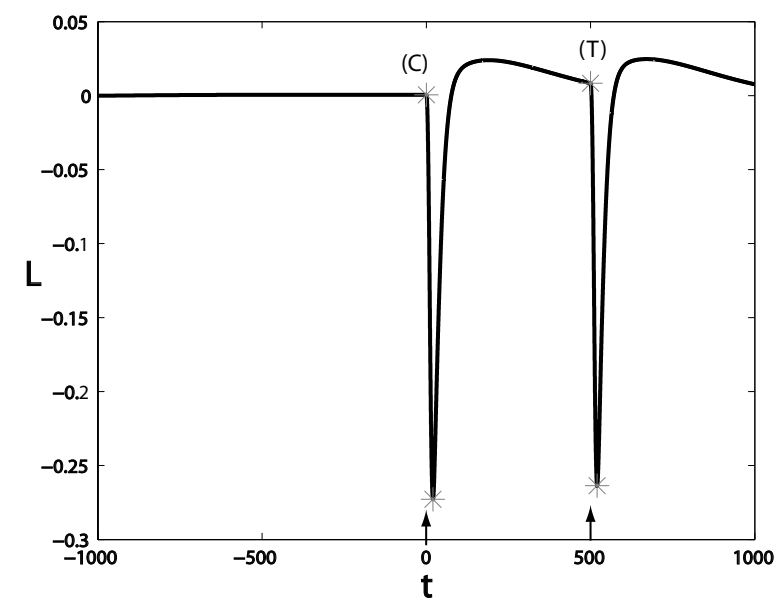

Fig. 5. Administration of CBs reduces the inhibition the principal population receives, causing a disruption of the gating and increasing the $\mathrm{T} / \mathrm{C}$ ratio to 1 . Parameters as in figure 4 except $C B_{\text {exo }}=1$. 


\section{Discussion}

We have explored one of the basic mechanisms underlying sensory gating, with a mixture of experiments and computational modelling. The minimal model we have developed uses a firing rate formalism combined with a phenomenological model of DSI that incorporates CB dynamics. Sensory gating in the model is highly dependent on the presence of a slow inhibitory process, namely the $\mathrm{GABA}_{\mathrm{B}}$ synapses connecting the basket cell population to the principal cell population. We show that gating can be abolished by the application of exogenous CBs, in a manner consistent with our experimental results.

A limitation of our model is that it can not deal with issues of spike timing or spike correlations. Additionally it fails to take into account both the spatially extended nature of the single cell, and indeed that of the CA3 hippocampal region. These are important when one recognises that endogenous CBs have a spatially local transient effect whereas exogenous CBs can have a more global one. One natural way to extend our work is to move away from population models to network models for the CA3 region of the hippocampus with synaptically interacting spiking neurons. We expect that the spike trains and LFPs obtained from such a biologically realistic model will be directly comparable to experimental data recorded using multi-electrode arrays and offer us the framework for exploring the effects of CBs on sensory gating in more detail. Experimental studies are ongoing to examine the mechanisms underlying the effects of endogenous and exogenous CBs on sensory gating.

\section{References}

[1] C. Bédard, H. Kröger and A. Destexhe, Modeling Extracellular Field Potentials and the Frequency-Filtering Properties of Extracellular Space, Biophys J 86 (2004) 1829-1842.

[2] P. C. Bickford and K. D. Wear, Restoration of sensory gating of auditory evoked response by nicotine in fimbria-fornix lesioned rats, Brain Res 705 (1995) 235-240.

[3] P. C. Bickford-Wimer, H. Nagamoto, R. Johnson, L. E. Adler, M. Egan and G. M. Rose, Auditory sensory gating in hippocampal neurons: A model system in rat, Biol Phychiatry 27 (1990) 183-192.

[4] F. Chaperon and M. H. Thiebot, Behavioral effects of cannabinoid agents in animals, Crit Rev Neurobiol 13 (1999) 243-281.

[5] N. M. W. J. de Bruin, B. A. Ellenbroek, E. L. J. M. Van Luijtelaar, A. R. Cools and K. E. Stevens, Hippocampal and cortical sensory gating in rats: Effects of quinpirole microinjections in nucleus accumbens core and shell, Neuroscience 105 (2001) 169-180. 
[6] N. M. W. J. de Bruin, E. L. J. M. Van Luijtelaar, A. R. Cools and B. A. Ellenbroek, Filtering Disturbances in Schizophrenic Patients. Gating of Auditory Evoked Potentials and Prepulse Inhibition of the Acoustic Startle Response Compared. Emphasis on the role of Dopamine, Current Neuropharmacol 1 (2003) 47-87.

[7] W. D. N. Dissanayake, M. Zachariou, C. A. Marsden and R. Mason, Abolition of sensory gating by the cannabinoid WIN55, 212-2 in the rat hippocampus, Psychopharmacol J 20 (2006) A45.

[8] H. M. Emrich, F. M. Leweke and U. Schneider. Towards a Cannabinoid Hypothesis of Schizophrenia: Cognitive Impairments Due to Dysregulation of the Endogenous Cannabinoid System, Pharmacol Biochem Behav 56 (1997) 803-807.

[9] K. A. Flach, L. E. Adler, G. A. Gerhardt, P. Bickford and R. J. MacGregor, Sensory gating in a computer model of the CA3 neural network of the hippocampus, Biol Psychiatry 40 (1996) 1230-1245.

[10] T. F. Freund, I. Katona and D. Piomelli, Role of endogenous cannabinoids in synaptic signalling, Physiol Rev 83 (2003) 1017-1066.

[11] K. M. Hershman, R. Freedman and P. C. Bickford, GABA $B$ antagonists diminish the inhibitory gating of auditory response in the rat hippocampus, Neurosci Lett 190 (1995) $133-136$.

[12] D. R. Lara, Inhibitory Deficit in Schizophrenia is Not Necessarily a GABAergic Deficit, Cell Mol Neurobiol 22 (2002) 239-247.

[13] C. L. Miller and R. Freedman, The activity of hippocampal interneurons and pyramidal cells during the response of the hippocampus to repeated auditory stimuli, Neuroscience 69 (1995) 371-381.

[14] K. A. Moxon, G. A. Gerhardt and L. E. Adler, Dopaminergic modulation of the P50 auditory-evoked potential in a computer model of the CA3 region of the hippocampus: its relationship to sensory gating in schizophrenia, Biol Cybern 88 (2003) 265-275.

[15] K. A. Moxon, G. A. Gerhardt, M. Gulinello and L. E. Adler, Inhibitory control of sensory gating in a computer model of the CA3 region of the hippocampus, Biol Cybern 88 (2003) 247-264.

[16] T. Ohno-Shosaku, T. Maejima and M. Kano, Endogenous cannabinoids mediate retrograde signals from depolarized postsynaptic neurons to presynaptic terminals, Neuron 26 (2001) $567-570$.

[17] V. Luntz-Leybman, P. C. Bickford and R. Freedman, Cholinergic gating of response to auditory stimuli in rat hippocampus, Brain Research 587 (1992) 130-136. 
[18] H. R. Wilson and J. D. Cowan, Excitatory and Inhibitory interactions in localized populations of model neurons, Biophys J 12 (1972) 1-24,

[19] R. I. Wilson and R. A. Nicoll, Endocannabinoid signaling in the brain, Science 296 (2002) 678-682.

[20] R. I. Wilson and R. A. Nicoll, Endogenous cannabinoids mediate retrograde signalling at hippocampal synapses, Nature 410 (2001) 1-4.

\section{Biographies}

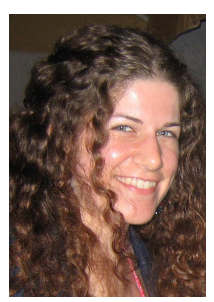

Margarita Zachariou received her BSc in Applied Mathematics from the University of Cyprus, Cyprus, in 2003. She is currently a Ph.D. student in the University of Nottingham, UK, under the supervision of Dr Stephen Coombes and Dr Rob Mason, in a joint degree between the Institute of Neuroscience and the School of Mathematical Sciences. Her research interests focus on investigating the neural network of the neurobiology of sensory gating using spiking and rate models. She is also applying computational and mathematical techniques to facilitate the analysis, presentation and interpretation of multiple electrode recordings of auditory evoked data.

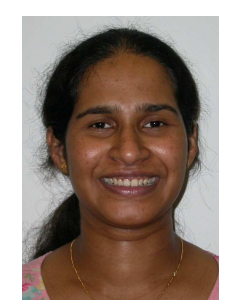

Dilshani Dissanayake received her MBBS from the Faculty of Medicine; University of Colombo, Sri Lanka in 2000. Following her post-graduate clinical training at the National hospital of Sri Lanka, she joined the Faculty of Medicine, University of Colombo, as a lecturer in Neurophysiology in 2002. She is currently reading for her Ph.D. on sensory gating deficits in rat model of schizophrenia, under the supervision of Dr Rob Mason and Prof Charles Marsden at the School of Biomedical Sciences, University of Nottingham, UK. Her research interests are on sensory processing deficits in neuropsychiatric diseases. 
Markus Owen received his Ph.D. in Applied Mathematics from the University of Warwick (UK) in 1997. He conducted post-doctoral work with Mark Lewis in the Department of Mathematics, University of Utah (USA), and subsequently spent five years as a Lecturer and Senior Lecturer in the Nonlinear and Complex Systems Group at Loughborough University (UK). He is currently a Reader in Applied Mathematics at the University of Nottingham (UK). His main research interests lie in the application of applied mathematics to the study of diverse biological systems, from plant biology to neuroscience.

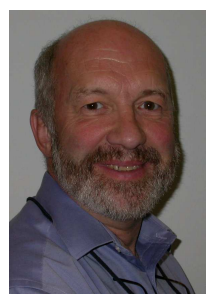

Rob Mason received his Ph.D. in Neuroscience from the University of Keele (UK) in 1976. He has conducted postdoctoral work at Leiden (Netherlands), Dalhousie and McMaster Universities (Canada). Currently he is Senior Lecturer in Neuroscience at the University of Nottingham Medical School (UK). His research interests are investigating interactions between neuronal ensembles in models of neurological and psychiatric disease states using multi-channel electrophysiological and MRI recording approaches.

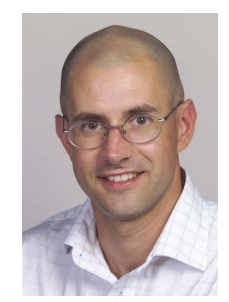

Stephen Coombes received his Ph.D. in Neurocomputing from King's College London (UK) in 1995. He conducted post-doctoral work with Colin Campbell in the Department of Engineering Mathematics, Bristol University (UK), and subsequently with Paul Bressloff in the Nonlinear and Complex Systems Group at Loughborough University (UK). He is currently a Reader in Mathematical Neuroscience at the University of Nottingham (UK), and holds an EPSRC Advanced Research Fellowship (2002-2007) to study synaptic travelling waves. His main research interests lie in the application of principles from nonlinear dynamics and statistical physics to the study of neural systems. 